\title{
La espirometría en preescolares
}

\author{
M. Pedrosa, S. Quirce
}

\section{Sr. Director:}

El asma es una enfermedad de distribución mundial que se estima que afecta a 300 millones de personas. La prevalencia de esta enfermedad va en aumento. Hay varias explicaciones que se han propuesto como responsables de este incremento en la prevalencia: exposición al humo del tabaco, contaminación del aire, cambios en la dieta, obesidad y las infecciones ${ }^{1}$. No obstante, el hecho de que haya una conciencia creciente del impacto que tienen las enfermedades respiratorias puede haber contribuido también a un mayor diagnóstico por parte de los profesionales sanitarios. Sin embargo, no todos los casos que se etiquetan como asma realmente lo son. Para evitar esto, las guías internacionales recomiendan que el diagnóstico de asma se base tanto en la presencia de síntomas como en medidas objetivas de una obstrucción variable al flujo aéreo ${ }^{2}$. La espirometría con prueba broncodilatadora tiene, por tanto, un elevado valor diagnóstico, aunque no siempre se realice, y esto es causa de muchos diagnósticos erróneos. Esto ha quedado demostrado en varios estudios en los que se ha reevaluado pacientes con un diagnóstico previo de asma y éste se excluyó en un $30-41 \%$ de los $\operatorname{casos}^{3-5}$.

El hecho de no realizar espirometrías es particularmente frecuente en el caso de niños preescolares ${ }^{6}$ puesto que se asume que esta población no es capaz de alcanzar los requerimientos de calidad propuestos para la aceptación de esta exploración. Sin embargo, los espirómetros actuales disponen de sistemas de incentivación y criterios modificados para la aceptabilidad y reproducibilidad, lo que permite realizar pruebas de función pulmonar en niños preescolares por personal entrenado.

Las indicaciones de realización de espirometría en niños con patología respiratoria incluyen la tos crónica, los sibilantes persistentes ${ }^{7} \mathrm{y}$ el diagnóstico y monitorización del asma ${ }^{8,9}$ y la fibrosis quística ${ }^{10,11}$.

Los parámetros que se valoran en la espirometría son $\mathrm{FEV}_{1}$, FVC y el cociente $\mathrm{FEV}_{1} / \mathrm{FVC}$, al igual que en adultos. El cociente $\mathrm{FEV}_{1} / \mathrm{FVC}$ es el parámetro más sensible y específico para identificar la obstrucción aérea. Estos parámetros se comparan con
Correspondencia:

Santiago Quirce

Servicio de Alergología

Hospital de La Paz

$\mathrm{P}^{\mathrm{o}}$ de la Castellana, 261

28046 Madrid

squirce@gmail.com 
los valores de referencia de la población normal, aunque son pocos los países que disponen de valores de referencia para la población infantil ${ }^{12-15}$. La variación en el $\mathrm{FEV}_{1}$ es altamente específica para el diagnóstico de asma en niños con síntomas inespecíficos de la vía respiratoria inferior $^{16}$. Además permite clasificar la gravedad del asma, y tiene utilidad para predecir las exacerbaciones ${ }^{17}$, la progresión de la enfermedad y el deterioro funcional de la calidad de vida. Una baja función pulmonar al nacimiento se asocia con un elevado riesgo de padecer asma a la edad de 10 años $^{18}$. Por este motivo se debe insistir en la realización de medidas de función pulmonar objetivas en los niños, ya que esto permite predecir el curso de la enfermedad.

Diversos autores han demostrado que los niños preescolares entre 2 y 6 años son capaces de realizar maniobras espirométricas aceptables en un 55-82\% de los $\operatorname{casos}^{19-21}$. A estos estudios hay que sumar los recientemente publicados en la revista Anales del Sistema Sanitario de Navarra por Olaguibel y $\mathrm{col}^{22}$. El estudio incluyó 114 niños preescolares sanos de los que 93 aceptaron participar en la realización de pruebas de función pulmonar. De ellos en 60 niños obtuvieron maniobras aceptables y reproducibles y en otros 16 se obtuvieron al menos dos maniobras aceptables, aunque no cumplían criterios de reproducibilidad. En los pacientes en los que las espirometrías no fueron exitosas el error más común fue la terminación prematura de la maniobra (39\% de los casos) y un pico flujo inadecuado (30\%). Este estudio demuestra que, a pesar de haber utilizado criterios de calidad exigentes y sobre una población sana no entrenada previamente, en el $59 \%$ de los niños preescolares se pueden obtener espirometrías forzadas de calidad. Por lo tanto, puesto que técnicamente la exploración funcional respiratoria es posible en niños preescolares y dadas las implicaciones diagnósticas, de seguimiento y pronósticas que tiene la medición de la función pulmonar desde edades tempranas, se debe fomentar la realización de exploración funcional en niños con patología respiratoria.

\section{BIBLIOGRAFÍA}

1. Eder W, Ege MJ, von Mutius E. The asthma epidemic. N Engl J Med 2006; 355: 2226-2235.

2. Initiative G. Global strategy for asthma management and prevention (GINA) 2014. Available at www.ginasthma.com.

3. Lindensmith J, Morrison D, Deveau C, Hernandez $\mathrm{P}$. Overdiagnosis of asthma in the community. Can Respir J 2004; 11: 111-116.

4. Aaron SD, Vandemheen KL, Boulet L-P, McIvor RA, Fitzgerald JM, Hernandez P, et al. Overdiagnosis of asthma in obese and nonobese adults. CMAJ 2008; 179: 1121-1131.

5. LuKs VP, Vandemheen KL, Aaron SD. Confirmation of asthma in an era of overdiagnosis. Eur Respir J 2010; 36: 255-260.

6. Dombkowski KJ, Hassan F, Wasilevich EA, Clark SJ. Spirometry use among pediatric primary care physicians. Pediatrics 2010; 126: 682687.

7. Constant C, Sampaio I, Negreiro F, Aguiar P, SiLVA AM, SAlgueIRo M et al. Respiratory disease screening in school-aged children using portable spirometry. J Pediatr (Rio J) 2011; 87: 123-130.

8. Holt EW, TAN J, Hosgood HD. The impact of spirometry on pediatric asthma diagnosis and treatment. J Asthma 2006; 43: 489-493.

9. Dundas I, Mckenzie S. Spirometry in the diagnosis of asthma in children. Curr Opin Pulm Med 2006; 12: 28-33.

10. Sanders DB, Rosenfeld M, Mayer-Hamblett N, Stamey D, RedDing GJ. Reproducibility of spirometry during cystic fibrosis pulmonary exacerbations. Pediatr Pulmonol2008; 43: 1142-1146.

11. Vilozni D, Bentur L, Efrati O, Minuskin T, Barak A, Szeinberg A et al. Spirometry in early childhood in cystic fibrosis patients. Chest 2007; 131: 356-361.

12. Pistelli F, Bottai M, Carrozzi L, Baldacci S, SiMONI M, Di PEDE F et al. Reference equations for spirometry from a general population sample in central Italy. Respir Med 2007; 101 : 814-825.

13. ChHabra SK, Vijayan VK, Rahman M, Mittal V, SINGH PD. Regression equations for spirometry in children aged 6 to 17 years in Delhi region. Indian J Chest Dis Allied Sci 2012; 54 : 59-63.

14. Stanojevic S, Wade A, Cole TJ, Lum S, Custovic A, Silverman M et al. Spirometry centile charts for young Caucasian children: the Asthma UK Collaborative Initiative. Am J Respir Crit Care Med 2009; 180: 547-552. 
15. Quanjer PH, Stanojevic S, Cole TJ, Baur X, Hall GL, CULver BH et al. Multi-ethnic reference values for spirometry for the 3-95-yr age range: the global lung function 2012 equations. Eur Respir J 2012; 40: 1324-1343.

16. Brouwer AFJ, VisSer CAN, Duiverman EJ, RoORdA RJ, BRAND PLP. Is home spirometry useful in diagnosing asthma in children with nonspecific respiratory symptoms? Pediatr Pulmonol 2010; 45: 326-332.

17. Fuhlbrigge AL, Kitch BT, Paltiel AD, Kuntz KM, Neumann PJ, Dockery DW, et al. FEV(1) is associated with risk of asthma attacks in a pediatric population. J Allergy Clin Immunol 2001; 107: 61-67.

18. Håland G, Carlsen KCl, Sandvik L, Devulapalli CS, Munthe-Kaas MC, Pettersen M, et al. Reduced lung function at birth and the risk of asthma at 10 years of age. N Engl J Med 2006; 355: 1682-1689.
19. Eigen H, Bieler H, Grant D, Christoph K, Terrill D, HeILMAn DK et al. Spirometric pulmonary function in healthy preschool children. Am J Respir Crit Care Med 2001; 163 (3 Pt 1): 619623.

20. Crenesse D, Berlioz M, Bourrier T, Albertini M. Spirometry in children aged 3 to 5 years: reliability of forced expiratory maneuvers. Pediatr Pulmonol 2001; 32: 56-61.

21. Aurora P, Stocks J, Oliver C, Saunders C, CastLE R, Chaziparasidis $\mathrm{G}$ et al. Quality control for spirometry in preschool children with and without lung disease. Am J Respir Crit Care Med 2004; 169: 1152-1159.

22. Olaguíbel Rivera J, Álvarez Puebla M, Vela Vizcaíno C, Cambra Contín K, Uribe San Martín M, de Esteban Chocarro B. Calidad de la espirometría en preeescolares. An Sist Sanit Navar 2014; 37: 81-89. 